\title{
Clinical Bedside Tools to Assess Systemic Sclerosis Vasculopathy: Can Digital Thermal Monitoring and Sublingual Microscopy Identify Patients With Digital Ulcers?
}

\author{
Tracy M. Frech ${ }^{1}$ (D), Zhining $\mathrm{Ou}^{2}$, and Angela P. Presson ${ }^{2}$
}

\begin{abstract}
Objective. Sublingual microscopy assesses systemic sclerosis (SSc) vasculopathy. Digital thermal monitoring (DTM) may identify patients at risk for digital ulcer (DU). The purpose of this analysis was to assess sublingual microscopy and DTM in SSc patients with and without previous DU in order to determine the utility of these clinical tools.

Methods. SSc registry patients with clinical data who had both DTM and sublingual microscopy on the same day were included in this cross-sectional analysis. DTM quantifies vascular reactivity index (VRI). Sublingual microscopy measures longitudinal red blood cell fraction (RBCfract) and perfused boundary region (PBR). We evaluated the pairwise association between VRI, RBCfract, and PBR in a monotonic relationship using Spearman rank correlation in the DU subset. Correlation coefficients $\left(\mathrm{r}_{\mathrm{s}}\right)$ and their $95 \%$ CIs were reported.

Results. Ninety patients were included; 29 had digital pits and/or active DU and 61 never had a DU. The only significant clinical feature associated with DU was modified Rodnan skin score $(P=0.003)$ with DU being higher. The VRI was lower in patients with DU $(P=0.01)$. The higher the RBCfract, the lower PBR $\left(\mathrm{r}_{\mathrm{s}}=-0.71,95 \% \mathrm{CI}-0.86\right.$ to $\left.-0.47, P<0.001\right)$. VRI was not associated with RBCfract or PBR $(P=0.24$ or 0.55 , respectively) in the patients with DU.

Conclusion. DTM is a useful tool for assessing SSc-DU. While sublingual microscopy measurements did not significantly correlate to VRI in patients with SSc-DU, a longitudinal study may be more helpful in capturing vasculopathy activity prior to possible irreversible damage.
\end{abstract}

Key Indexing Terms: microscopy, Raynaud phenomenon, systemic sclerosis

Systemic sclerosis (SSc) is an autoimmune disease characterized by progressive vascular damage (vasculopathy) with resultant fibrosis and immune dysregulation. The most common manifestation of vasculopathy in SSc is Raynaud phenomenon (RP), which is a triphasic color change of the digits that occurs in response to emotional stress and cold temperature, and is present in almost all patients with SSc. ${ }^{1}$ Whereas RP is easily visualized on a skin assessment, the vasculopathy of SSc is also internal and multiorgan. As such, end-organ damage assessment tools are commonly used to define SSc vasculopathy. For example,

This work was supported by awards from the National Institutes of Health (NIH; K23AR067889) and the U.S. Department of Veterans Affairs (I01 CX001183). This investigation was supported by the University of Utah Population Health Research (PHR) Foundation, with funding in part from the National Center for Research Resources and the National Center for Advancing Translational Sciences, NIH, through Grant UL1TR002538.

${ }^{I}$ T.M. Frech, MD, MS, Department of Internal Medicine, Division of Rheumatology, University of Utah; ${ }^{2} Z$. Ou, MS, A.P. Presson, PhD, MS, Department of Internal Medicine, Division of Epidemiology, University of Utah, Salt Lake City, Utah, USA.

The authors have no conflict of interest relevant to this article. Address correspondence to Dr. T.M. Frech, 1900 E 30 N, SOM 4b200, Salt Lake City,UT 84132,USA.Email: tracy.frech@hsc.utah.edu. Accepted for publication January 15, 2021. right heart catheterization is used to diagnose pulmonary arterial hypertension (PAH). Nailfold videocapillaroscopy is the most commonly used tool to diagnose RP related to SSc and has features that correlate to PAH. In severe and prolonged RP episodes, ischemic digital ulcers (DUs) can occur in this patient population, and are associated with a decrease in capillaroscopy density on nailfold capillaroscopy. ${ }^{2}$ Similar to nailfold capillaroscopy, tools that can be used in the clinical setting to identify patients at risk for DU, but can also help clarify pathogenesis, are needed to improve management and potentially enrich clinical trials for this indication. ${ }^{3}$

Our group has previously reported on the ease of using digital thermal monitoring (DTM) and sublingual microscopy for clinical vasculopathy assessment in SSc. ${ }^{45,6}$ DTM (VENDYS, Endothelix Inc.) is a procedure that quantifies the thermal response of the fingers in response to ischemia-induced blood pressure cuff occlusion by a single vascular reactive index (VRI) measurement. The rationale is that a VRI measurement of temperature rebound is a useful assessment of vasculopathy attributed to RP. Our group has reported that VRI correlates to flow-mediated dilation and thus may be useful for DU assessment. $^{5}$

For mechanistic vasculopathy assessment, our group and others ${ }^{7,8}$ have used sublingual microscopy. Sublingual microscopy (GlycoCheck, Microvascular Health Solutions Inc.) provides an 
automated measure of systemic microvascular perfusion (longitudinal red blood cell fraction [RBCfract]) and glycocalyx penetrability (perfused boundary region $[\mathrm{PBR}])$. Mechanistically, the glycocalyx is an estimate of vascular health. Our group has shown that this GlycoCheck system can distinguish SSc patients' vasculopathy from healthy controls. ${ }^{6}$ The purpose of this project was to look at these 2 bedside vasculopathy tools in patients with SSc to determine if a single assessment of temperature rebound and vascular health vs longitudinal assessment is needed for DU identification.

\section{METHODS}

Patients who consented and were enrolled in the Utah SSc registry (institutional review board approval no. 38705) were included in this cross-sectional analysis. Sociodemographics and SSc disease features, including modified Rodnan skin score (mRSS), autoantibody status, and DU status were recorded. Inclusion criteria required DTM and sublingual microscopy procedures to be performed on the same day of routine clinical care. Measurements were performed in a quiet room at a controlled ambient temperature. Patients abstained from exercise, tobacco, and caffeine for a minimum of 12 hours prior to the assessment.

We summarized demographics and clinical outcomes of interest using median, IQR, and range for continuous variables. We reported counts and percentages for categorical variables. Wilcoxon rank-sum exact test and
Fisher exact test were used to compare variables between DU groups. We evaluated the pairwise association between VRI, RBCfract, and PBR in a monotonic relationship using Spearman rank correlation in the DU subset. We reported the correlation coefficients $\left(\mathrm{r}_{\mathrm{s}}\right)$, their $95 \% \mathrm{CIs}$, and the asymptotic $P$ values. Statistical significance was assessed at the 0.05 level. All tests were 2 -sided. Statistical analyses were implemented using R v3.6.0 (R Core Team).

\section{RESULTS}

Ninety patients with SSc completed DTM and sublingual microscopy at their clinic visit. Of these participants, 29 had tender digitals pits (that, based on the assessment of the treating physician, was representative of a previous ischemic DU) and/ or active ischemic DU; 61 never had a DU (Table 1). These patients were $84.4 \%$ female, $92.2 \%$ White, $10 \%$ current smokers, and $87 \%$ SSc-specific autoantibody positive. At the time of assessment, 91\% were on vasodilators for RP. The only significant clinical feature associated with the presence of DU was a higher mRSS (median 7.0 vs 3.5, $P=0.003$; Table 1 ).

The VRI captured by DTM was lower in patients with a DU (median 0.6 vs $0.9, P=0.01$; Table 1 ). The sublingual microscopy RBCfract and PBR, both of which indicate vascular health, were monotonic associated $(P<0.001)$ such that the higher

Table 1. Clinical features of 90 patients with SSc assessed with digital thermal monitoring and sublingual microscopy.

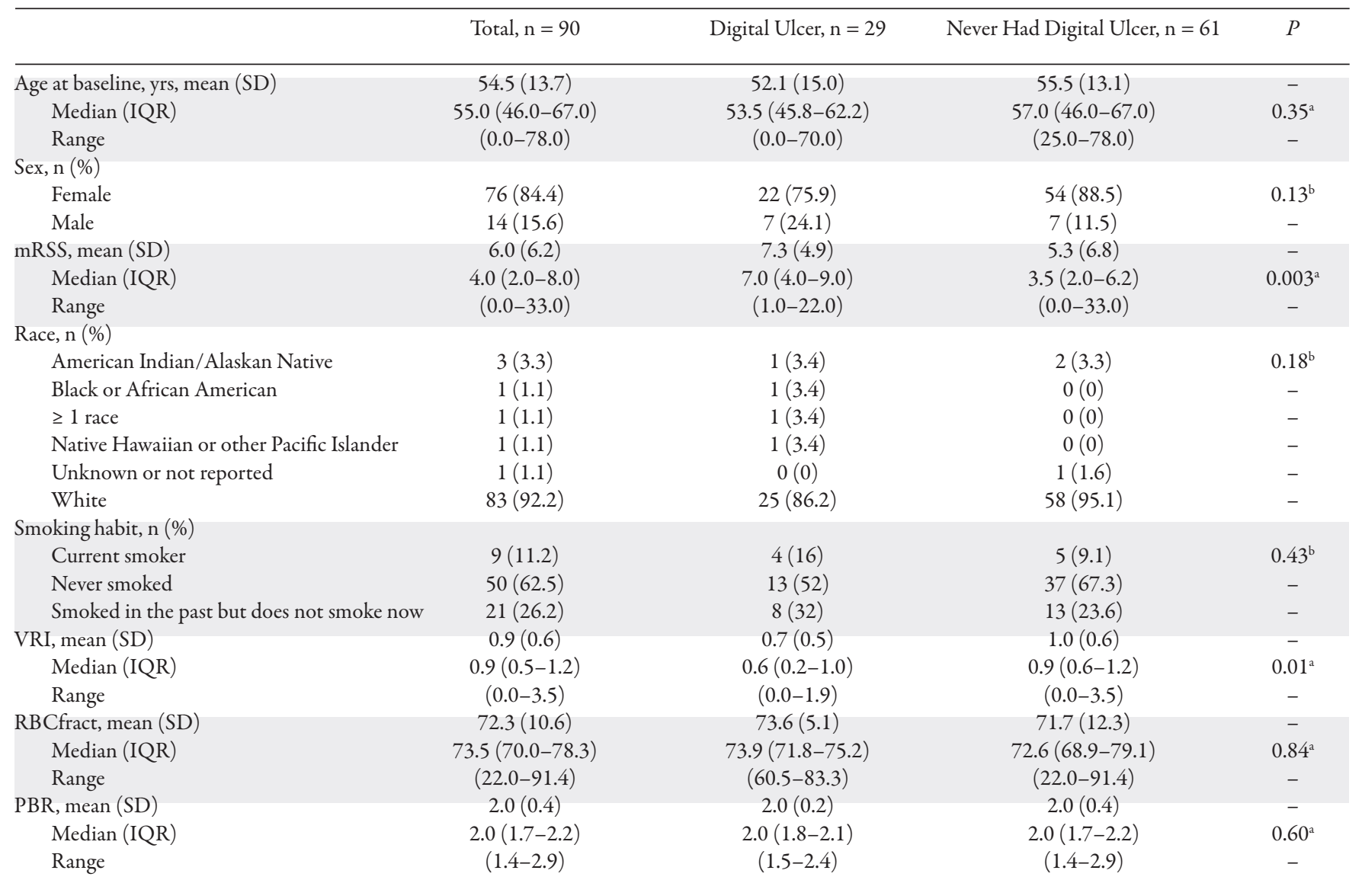

Missing values: age at baseline $=1 ; \mathrm{mRSS}=1 ;$ smoking habit $=10 .{ }^{\mathrm{a}}$ Wilcoxon rank-sum exact test. ${ }^{\mathrm{b}}$ Fisher exact test. $\mathrm{mRSS}$ : modified Rodnan skin score; PBR: perfused boundary region; RBCfract: red blood cell fraction; VRI: vascular reactivity index. 
Table 2. Spearman rank correlation, $95 \% \mathrm{CI}$, and $P$ values for 29 patients with digital ulcers.

\begin{tabular}{lccc}
\hline & VRI & RBCfract & PBR \\
\hline VRI & - & $\mathrm{r}_{\mathrm{s}}=0.23(95 \% \mathrm{CI}$ & $\mathrm{r}_{\mathrm{s}}=0.12(95 \% \mathrm{CI}$ \\
& & -0.15 to 0.55$)$ & -0.26 to 0.46$)$ \\
RBCfract & $P=0.24$ & - & $\mathrm{r}_{\mathrm{s}}=-0.71$ \\
& & & $(-0.86$ to -0.47$)$ \\
PBR & $P=0.55$ & $P<0.001$ & - \\
\hline
\end{tabular}

PBR: perfused boundary region; RBCfract: red blood cell fraction; VRI: vascular reactivity index.

RBCfract, the lower PBR ( $r_{s}=-0.71,95 \%$ CI -0.86 to -0.47 ; Table 2). Among the 29 patients with DU, VRI was not associated with RBCfract or PBR $(P=0.24$ or 0.55 , respectively).

\section{DISCUSSION}

Clinical assessments that can be used to identify and quantify the mechanism of vasculopathy in patients with SSc are important to inform management and possibly enrich clinical trials for DU. Specifically, tools are needed to accurately characterize disease pathogenesis as it progresses from early microvascular changes with endothelial cell dysfunction, to the activation of mechanisms promoting their transition into myofibroblasts and fibrosis; these will allow the initiation of therapeutics that can prevent ischemia and irreversible damage to the skin. ${ }^{9}$ Our cross-sectional study further supports the concept that aberrant endothelial response to ischemia and inadequate dermal vasodilation in SSc is a risk for DUs. ${ }^{10}$ We have shown that DTM effectively quantified vascular damage, and thus, is a useful tool for assessing DUs in the SSc population. It is possible that this measure of temperature rebound could also be used longitudinally to assess VRI response to vasodilators.

Sublingual microscopy provides an estimate of systemic vascular health. Deterioration of the endothelial glycocalyx, a protective carbohydrate-rich layer lining the luminal surface of the endothelium, plays a critical role in vascular barrier dysfunction and leukocyte adhesion. ${ }^{11,12}$ Our data suggest that a single measurement of sublingual variables of RBCfract and PBR was inadequate for identifying patients with DUs and did not significantly correlate with VRI. A longitudinal, multicenter study of these sublingual microscopy variables may be more helpful in capturing vasculopathy activity prior to the possible irreversible damage that is perhaps better captured by DTM. Subject preparation for sublingual microscopy needs to be defined. Our data are consistent with other studies that highlight the association of a higher mRSS with DUs. ${ }^{13}$ The important concept of concurrently capturing activity and damage indices in SSc is supported by our data. ${ }^{14}$

In conclusion, the clinical practice of quantifying vasculopathy is imperative in SSc and is supported by this pilot study at a single center, supporting the feasibility of DTM and sublingual microscopy. The sensitivity of change and validity of these measurement tools will require a longitudinal study. Additionally, a larger, more diverse population that can stratify patients by clinical features such SSc-specific autoantibody subtype may be informative. Concurrent use of patient-reported outcomes that quantify the severity of RP and resultant DUs are needed. Nonetheless, this study shows that there is now a stronger case for the rheumatologist's use of automated vasculopathy assessment tools that can easily be implemented in the clinical setting.

\section{REFERENCES}

1. Hughes M, Herrick AL. Raynaud's phenomenon. Best Pract Res Clin Rheumatol 2016;30:112-32.

2. Hughes M, Allanore Y, Chung L, Pauling JD, Denton CP, Matucci-Cerinic M. Raynaud phenomenon and digital ulcers in systemic sclerosis. Nat Rev Rheumatol 2020;16:208-21.

3. Cutolo M, Smith V, Furst DE, Khanna D, Herrick AL. Points to consider-Raynaud's phenomenon in systemic sclerosis. Rheumatology 2017;56 Suppl 5:v45-8.

4. Thomas J, Radic M, Tucker JR, Overbury R, Frech T. Raynaud's phenomenon in systemic sclerosis: does digital thermal monitoring correlate to specific nailfold videocapillaroscopy abnormalities? J Rheumatol 2021;48:247-50.

5. Frech TM, Murtaugh MA. Non-invasive digital thermal monitoring and flow-mediated dilation in systemic sclerosis. Clin Exp Rheumatol 2019;37 Suppl 119:97-101.

6. Machin DR, Gates PE, Vink H, Frech TM, Donato AJ. Automated measurement of microvascular function reveals dysfunction in systemic sclerosis: a cross-sectional study. J Rheumatol. 2017;44:1603-11.

7. Miranda S, Armengol G, Le Besnerais M, Levesque H, Benhamou Y. New insights into systemic sclerosis related microcirculatory dysfunction by assessment of sublingual microcirculation and vascular glycocalyx layer. Results from a preliminary study. Microvasc Res 2015;99:72-7.

8. Sha M, Griffin M, Denton CP, Butler PE. Sidestream dark field (SDF) imaging of oral microcirculation in the assessment of systemic sclerosis. Microvasc Res 2019;126:103890.

9. Cutolo M, Soldano S, Smith V. Pathophysiology of systemic sclerosis: current understanding and new insights. Expert Rev Clin Immunol 2019;15:753-64.

10. Bruni C, Frech T, Manetti M, Rossi FW, Furst DE, De Paulis A, et al. Vascular leaking, a pivotal and early pathogenetic event in systemic sclerosis: should the door be closed? Front Immunol 2018;9:2045.

11. Alphonsus CS, Rodseth RN. The endothelial glycocalyx: a review of the vascular barrier. Anaesthesia 2014;69:777-84.

12. Mulivor AW, Lipowsky HH. Role of glycocalyx in leukocyte-endothelial cell adhesion. Am J Physiol Heart Circ Physiol 2002;283:H1282-91.

13. Pearson DR, Werth VP, Pappas-Taffer L. Systemic sclerosis: current concepts of skin and systemic manifestations. Clin Dermatology 2018;36:459-74.

14. Nikpour M; Scleroderma Clinical Trials Consortium Damage Index (SCTC-DI) Working Group. Response to: 'Differentiating disease activity from damage in systemic sclerosis: it's still early days!' by Jain and Sharma. Ann Rheum Dis 2020;79:e99. 